\title{
A Downsampled SAR-BM3D Despeckling Approach for Single-Look SAR Images in High Resolution
}

\author{
Wuchao Wang, Xiaolin Liu, Wenlong Zhang \\ National University of Defense Technology, Changsha, China \\ Email: slashsuper@163.com
}

How to cite this paper: Wang, W.C., Liu, X.L. and Zhang, W.L. (2016) A Downsampled SAR-BM3D Despeckling Approach for Single-Look SAR Images in High Resolution. Journal of Computer and Communications, 4, 126-131.

http://dx.doi.org/10.4236/jcc.2016.415012

Received: October 28, 2016

Accepted: November 25, 2016

Published: November 28, 2016

\begin{abstract}
SAR-BM3D is one of the state of the art despeckling algorithms for SAR images. However, when tackling with high resolution SAR images, it often has an unsatisfying despeckling performance in the homogeneous smooth regions, together with a high time complexity. In this paper, a novel downsampled SAR-BM3D despeckling approach combined with edge compensation is proposed. The proposed algorithm consists of two steps. First, despeckle the image which is a downsampled version of original image with SAR-BM3D. Then, compensate edges in each level when upsampling. This approach not only utilizes the good ability of feature preservation, but also improves performance of smoothing homogenous regions. When it comes to high resolution SAR images, the efficiency can be raised by six to seven times, compared to original SAR-BM3D. Experiments on simulated and real SAR images show that the proposed method reaches a high level in terms of visual quality and act more efficiently.
\end{abstract}

\section{Keywords}

Despeckling, SAR-BM3D, Downsampling, High Resolution, Synthetic Aperture Radar (SAR)

\section{Introduction}

Due to its all-weather and all-time capability, SAR has being utilized in various applications in both civilian and military. However, speckle which degrades the radiometric quality of data generates because of its imaging mechanism. For further analysis and interpretation, despeckling is necessary and significant for preprocessing.

In past decades, lots of classic despeckling methods were proposed, such as Lee, Kuan, Frost and hard/soft threshold approach based on the wavelet transform [1]. In 2005, inspired by the local filtering, Buades et al. proposed non-local means method 
(NLM) [2], where similar patches of the target patch are searched all over the image and then the pixels are averaged according to the similarity weights of the pair patches. Deledalle et al. proposed a probabilistic patch-based (PPB) approach [3]. It extends the NLM filter to the domain of SAR images by exploiting its connections to the weighted maximum likelihood estimator (WMLE), which reached the state of the art performance at that time. To improve the accuracy of similarity measure, many methods are presented [5] [6] [7] based on nonlocal patch matching. One of the state of the art algorithms is BM3D [7] by Dabov et al. BM3D combines nonlocal patch matching with a wavelet representation. It outperforms NLM especially in details keeping.

Parrilli et al. adapted the BM3D filter to SAR images and proposed the SAR-BM3D method [8], which considers two main modifications: 1) the patch similarity measure is calculated according to PPB [3]; 2) LLMMSE estimator is used to replace the hardthresholding and Wiener filtering.

Adaptive search size selecting, probabilistic early termination and look-up table are adapted in FANS (Fast adaptive nonlocal SAR despeckling) [9] to speed up SAR-BM3D. FANS indeed reduce time complexity by $1 / 10$ of SAR-BM3D. It can keep details as well as SAR-BM3D in spite of losing some tiny ones. The performance in smoothing is not stable (sometimes over-smooth resulting artifacts). To get better image quality in homogenous regions, literature [10] and [11] both introduce classification. Both of them improve the image quality compared to SAR-BM3D to some degree.

Despeckling for SAR images aims to smoothing the homogenous regions and preserving details (like edges and isolated targets) as far as possible. Although SAR-BM3D has a strong capability of feature keeping, insufficient smoothing in homogenous regions and a high time complexity restrict its application. When processing single-look real SAR image in high resolution, SAR-BM3D cannot take both effect and efficiency into consideration, and achieve satisfying results, so as the variations of SAR-BM3D.

\section{Proposed Despeckling Strategy}

To adapt SAR-BM3D to single-look real SAR-BM3D in high resolution, this paper presents a method based on downsampled SAR-BM3D combined with edge compensation. The approach proposed here is a strategy which is a combination of BM3D and FANS. So, we don't present details of BM3D and FANS here. For more, please refer to [8] [9].

This proposed method has two major advantages:

1) Downsampling turns single-look image into a multilook version, which facilitates smoothing homogenous regions while SAR-BM3D holding the feature preservation capability.

2) Downsampling decreases the size of image, the efficiency of SAR-BM3D increases proportionally to square of sampling coefficient.

\subsection{Downsampled SAR-BM3D}

Downsampling works like multilook processing. After conducting downsampling with 
sampling coefficient equaling $k$ (power of 2 ), the image size reduces by $1 / k^{2}$ (given original size equals 1). Theoretically, the ENL (equivalent noise level) [11] will increases by $k^{2}$ (given original ENL equals 1). Obviously, the bigger $k$ is, the more efficient downsampled SAR-BM3D is. However, an excessive $k$ will result in over smoothing. It is necessary to select $k$ in terms of image resolution. Here, $k=4$ is recommended for high resolution SAR images while $k=2$ for low resolution ones.

During the conducting of SAR-BM3D after downsampling, a significant parameter should be taken into consideration, that is, $L$ which stands for ENL [11], to improve the performance of SAR-BM3D in smoothing homogenous regions. A relatively small $\mathrm{L}$ that equals to the root of the theoretical ENL is chosen experimentally in order to make the SAR-BM3D obtain a satisfying balance between smoothing (in homogenous regions) and details preservation.

$$
L=\sqrt{E N L_{\text {down }}}
$$

\subsection{Edge Compensation}

Downsampled SAR-BM3D can avoid the deficient smoothing to some extent. However, some detail information is lost after downsampling and upsampling. The whole image seems blurred and suffers from contrast degradation especially in the regions where edges and isolated targets exist. Therefore, edge compensation based on FANS is conducted as the complementary for downsampled SAR-BM3D. FANS is a faster version of BM3D. Figure 1 shows details of edge compensation, given that $k=2$. It works like restoring image with Laplacian pyramid. Filtered original image by FANS is used to approximate the result by SAR-BM3D.

\section{Experimental Results}

To justify the validity and efficiency of the proposed approach, we conducted experiments on a simulated image and a real-world single-look SAR images, each of which is $512 * 512$. Figure $2(\mathrm{a})$ is a corrupted Barbara image with a multiplicative speckle noise, of which the equivalent number of looks $L=1$; Figure 3(a) is a real single-look SAR image identified as Toulouse sensed by RAMSES and provided by the CNES. We

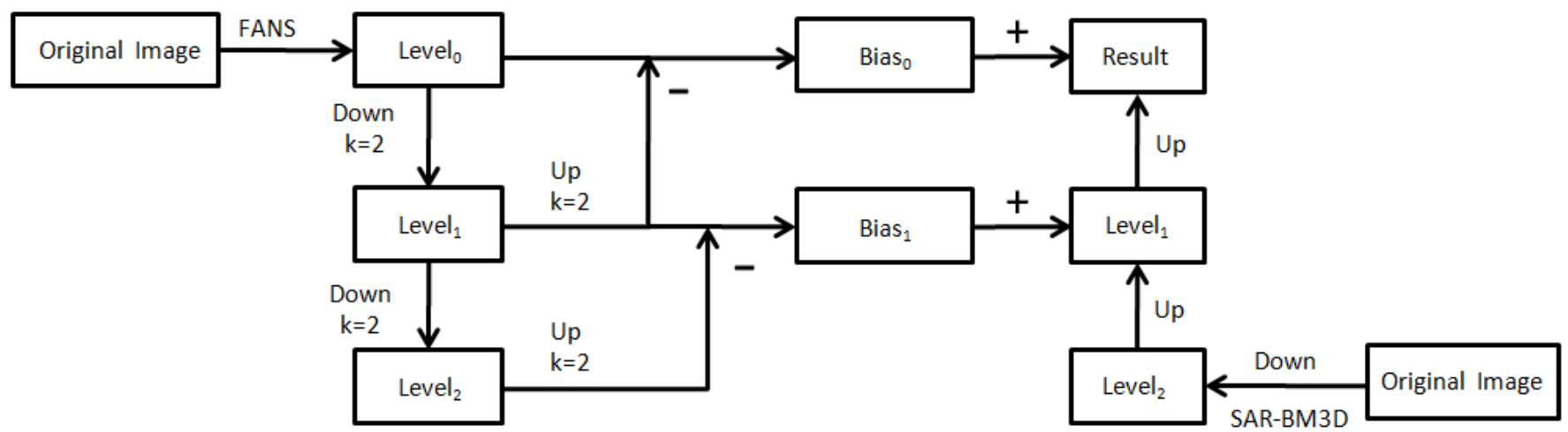

Figure 1. Flow chart of the proposed approach. 


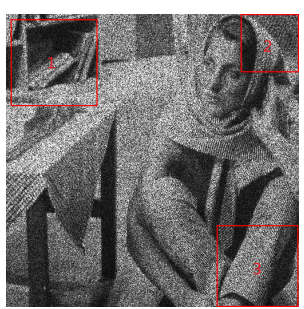

(a)

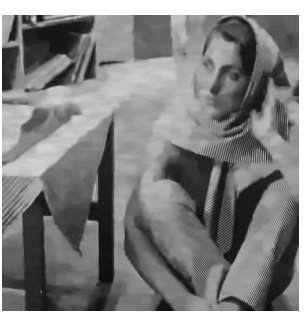

(b)

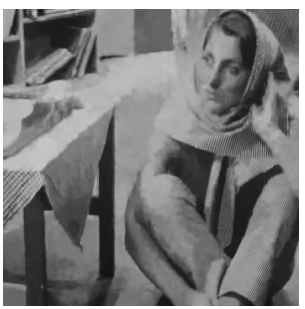

(c)

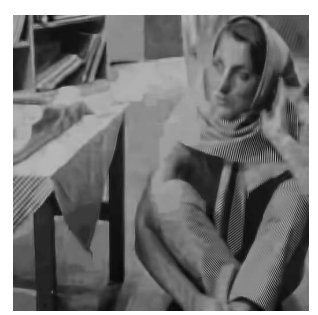

(d)

Figure 2. (a) Noisy image (Barbara); (b) FANS denoised image; (c) SAR-BM3D denoised image; (d) The proposed approach denoised image.
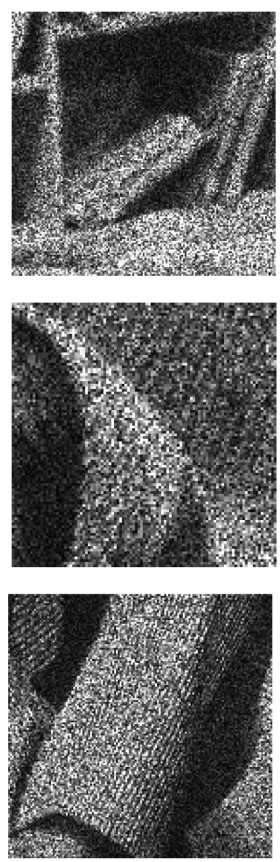

(a)
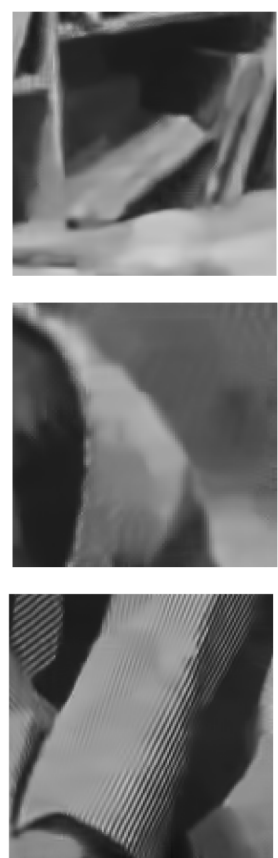

(b)
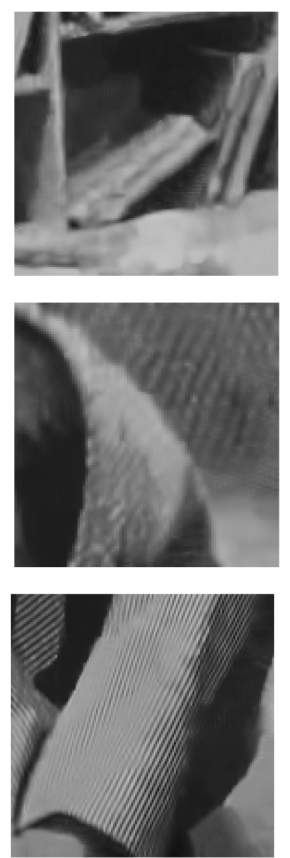

(c)
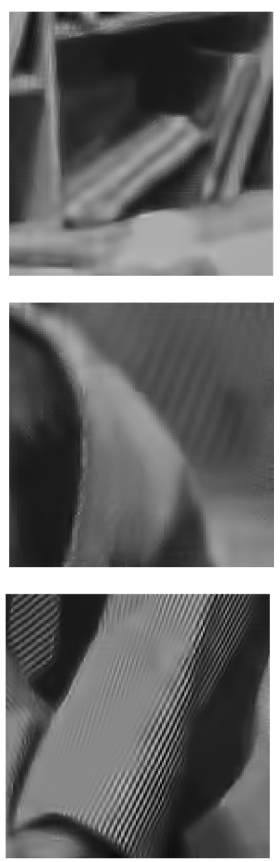

(d)

Figure 3. Details of two regions (Areas 1 and 2 in Figure 2(a)) in Figure 2.

compare the proposed method with SAR-BM3D and FANS from the perspectives of quality and efficiency. The algorithm complexity is measured by CPU (Intel(R) Xeon(R)) time on a $2.4 \mathrm{GHz} 64$ bit desktop computer with 32G memory.

Parameters of SAR-BM3D and FANS are referred to literature [8] and [9] respectively. In the proposed method, the sampling coefficient $\mathrm{k}$ is set as 2 due to low resolution of these two images, and. $L$ is 2 according to Equation (1).

Figure 2 shows the results of the simulated SAR image named Barbara. First, in homogenous regions in Figure 2(d) can be recognized smoother with naked eye. Then, it can be concluded that the feature preservation capability of the proposed method outperforms FANS and approximates BM3D through Figure 3. The outline of books in Figure 3(d) looks sharper and more satisfying than in Figure 3(b) and Figure 3(c). But the some tiny texture in the top right-hand corner and trousers in the bottom right-hand corner loses in Figure 2(d), while BM3D keeps them. It's quite obvious that 
the details missed in Figure 2(d) are of low contrast. They may get blurred in downsampling. However, when it comes to real SAR images, details (especially edge and isolated points which are always targets we care) are of high contrast. It seems that the proposed method will perform better in processing real SAR data.

Then, Figure 4 shows the results on real single-look SAR image. Even though Toulouse is not of high resolution, the proposed approach works well on it. BM3D does not perform well in smoothing homogenous regions, while FANS over-smooth some regions resulting artifacts resembling oil painting. Apparently, the proposed approach outperforms both of them in smoothing. Details, especially isolated point targets, are protected in the proposed approach pretty well.

Table 1 shows the average time (100 realizations) of the images processed by the three algorithms. Obviously, the proposed method is just about three rather than seven times faster than original SAR-BM3D. Because sampling coefficient $k$ equals 2 . The images are of low resolution, we cannot assign $k$ a bigger number according to Section 2.1.

The proposed approach is aimed at single-look high resolution real SAR images. Due to copyright, however, we cannot display results on real high resolution data. Experiments on $2000 * 2000$ high resolution single-look real-world SAR images are conducted. We assign sampling coefficient as 4 . Let $L$ equals 4 . Results show that the proposed approach is superior toBM3D and FANS in smoothing (homogenous regions) and details preservation and obtains a better visual effect. Table 2 shows time for despeckling these real data. It proves that the proposed method is 7 times faster than original SAR-BM3D.

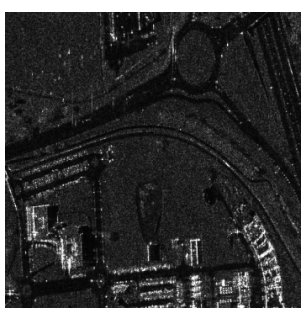

(a)

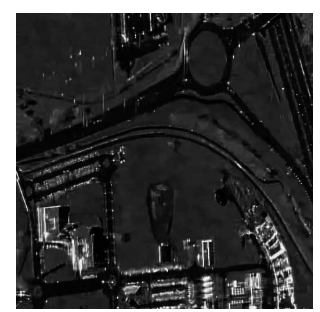

(b)

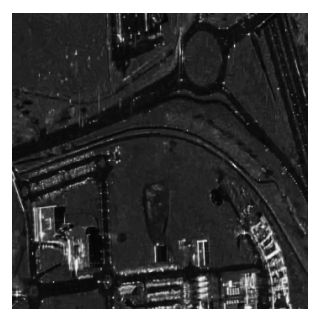

(c)

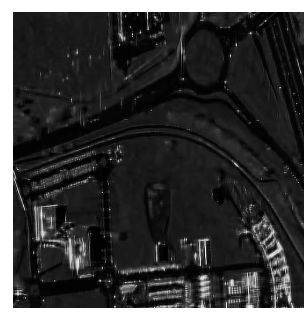

(d)

Figure 4. (a) Original SAR image (Toulouse $\odot$ DGA $\odot$ ONERA); (b) FANS denoised image; (c) SAR-BM3D denoised image; (d) The proposed approach denoised image.

Table 1. CPU Time for Barbara and Toulouse over 100 realizations.

\begin{tabular}{cccc}
\hline & FANS & BM3D & Proposed \\
\hline Barbara & $7.3(\mathrm{~s})$ & $83.6(\mathrm{~s})$ & $27.6(\mathrm{~s})$ \\
Toulouse & $7.3(\mathrm{~s})$ & $83.6(\mathrm{~s})$ & $28.9(\mathrm{~s})$ \\
\hline
\end{tabular}

Table 2. CPU Time for single-look SAR images $\left(2000^{\star} 2000\right)$ in high resolution over 50 realizations.

\begin{tabular}{ccc}
\hline FANS & BM3D & Proposed \\
\hline $113(\mathrm{~s})$ & $1270(\mathrm{~s})$ & $190(\mathrm{~s})$ \\
\hline
\end{tabular}




\section{Conclusion}

In general, downsampled SAR-BM3D with edge compensation approach gives satisfying results with much less running time on single-look SAR images in high resolution. Experiments justify its validity and efficiency on low-resolution single-look SAR images. Even though some problems still exist, namely that tiny artifacts appear in high-resolution SAR image results, further filtering on bias map will be researched in the following work.

\section{References}

[1] Argenti, F., Lapini, A., Bianchi, T., et al. (2013) A Tutorial on Speckle Reduction in Synthetic Aperture Radar Images. IEEE Geoscience and Remote Sensing Magazine, 1, 6-35. http://dx.doi.org/10.1109/MGRS.2013.2277512

[2] Buades, A., Coll, B. and Morel, J.M. (2005) A Non-Local Algorithm for Image Denoising. IEEE Computer Society Conference on Computer Vision and Pattern Recognition (CVPR'05), 2, 60-65.

[3] Deledalle, C.A., Denis, L. and Tupin, F. (2009) Iterative Weighted Maximum Likelihood Denoising with Probabilistic Patch-Based Weights. IEEE Transactions on Image Processing, 18, 2661-2672. http://dx.doi.org/10.1109/TIP.2009.2029593

[4] Louchet, C. and Moisan, L. (2011) Total Variation as a Local Filter. SIAM Journal on Imaging Sciences, 4, 651-694. http://dx.doi.org/10.1137/100785855

[5] Yang, X. and Clausi, D.A. (2009) Structure-Preserving Speckle Reduction of SAR Images Using Nonlocal Means Filters. 16th IEEE International Conference on Image Processing (ICIP), 2985-2988.

[6] Zhong, H., Zhang, J. and Liu, G. (2014) Robust Polarimetric SAR Despeckling Based on Nonlocal Means and Distributed Lee Filter. IEEE Transactions on Geoscience and Remote Sensing, 52, 4198-4210. http://dx.doi.org/10.1109/TGRS.2013.2280278

[7] Dabov, K., Foi, A., Katkovnik, V., et al. (2007) Image Denoising by Sparse 3-D TransformDomain Collaborative Filtering. IEEE Transactions on Image Processing, 16, 2080-2095. http://dx.doi.org/10.1109/TIP.2007.901238

[8] Parrilli, S., Poderico, M., Angelino, C.V., et al. (2012) A Nonlocal SAR Image Denoising Algorithm Based on LLMMSE Wavelet Shrinkage. IEEE Transactions on Geoscience and Remote Sensing, 50, 606-616. http://dx.doi.org/10.1109/TGRS.2011.2161586

[9] Cozzolino, D., Parrilli, S., Scarpa, G., et al. (2014) Fast Adaptive Nonlocal SAR Despeckling. IEEE Geoscience and Remote Sensing Letters, 11, 524-528.

http://dx.doi.org/10.1109/LGRS.2013.2271650

[10] Liu, S.J., Wu, G.Q., Zhang, X.Z., et al. (2016) SAR Image Despeckling via the Classification-Based Non-Local Clustering. Systems Engineering and Electronics, 38, 511-556

[11] Gragnaniello, D., Poggi, G., Scarpa, G., et al. (2015) SAR Despeckling Based on Soft Classification. IEEE International Geoscience and Remote Sensing Symposium (IGARSS), 23782381 . 
Submit or recommend next manuscript to SCIRP and we will provide best service for you:

Accepting pre-submission inquiries through Email, Facebook, LinkedIn, Twitter, etc. A wide selection of journals (inclusive of 9 subjects, more than 200 journals)

Providing 24-hour high-quality service

User-friendly online submission system

Fair and swift peer-review system

Efficient typesetting and proofreading procedure

Display of the result of downloads and visits, as well as the number of cited articles

Maximum dissemination of your research work

Submit your manuscript at: http://papersubmission.scirp.org/

Or contact jec@scirp.org 